\title{
ON RANDOM WALKS WITH A REFLECTING BARRIER
}

\author{
BY \\ SIDNEY C. PORT
}

1. Introduction. Let $\left\{X_{n}\right\}$ be a sequence of independent, identically distributed random variables. One Markov process associated with the $X_{n}$ which has been intensively investigated is the free random walk,

$$
S_{n}^{\prime}=S_{0}^{\prime}+X_{1}+\cdots+X_{n}=S_{0}^{\prime}+S_{n} .
$$

In this paper we shall investigate another Markov process, $T_{n}$, which is also associated with the $X_{n}$. This $T_{n}$ process is defined as follows:

$T_{0}$ is an arbitrary nonnegative random variable independent of the $X_{n}$, and for $n>0$,

$$
T_{n}=\left(X_{n}+T_{n-1}\right)^{+},
$$

where for any quantity $x$ we have $x^{+}=x$ if $x>0$ and $x^{+}=0$ if $x \leqq 0$. The $T_{n}$ process behaves like the $S_{n}^{\prime}$, with the vital difference that the origin acts as a reflecting barrier on the left. This process was introduced originally as an auxiliary device in the investigation of $M_{n}$, the maximal partial sum amongst $\left(S_{1}, S_{2}, \cdots, S_{n}\right)$, because of the familiar relation

$$
P\left(M_{n}^{+} \leqq x\right)=P\left(T_{n} \leqq x \mid T_{0}=0\right)
$$

(see Lemma 2.1). Previously, the $T_{n}$ were investigated by Spitzer in [6] and [7], and the results we obtain here will be extensions of those of Spitzer. However, our methods are quite different from those of Spitzer.

Before proceeding further, let us adopt some notation which we shall use throughout the remainder of this paper. Let $x \geqq 0$, and let $A$ be a Borel set on the nonnegative axis. Then, if $n>0$,

$$
\begin{aligned}
P_{n}(x ; A) & =P\left(T_{n} \in A \mid T_{0}=x\right), \\
{ }_{0} P_{n}(x ; A) & =P\left(T_{n} \in A ; T_{i} \neq 0, \quad 1 \leqq i<n \mid T_{0}=x\right),
\end{aligned}
$$

while for $n=0$ we have

$$
P_{0}(x ; A)=\delta_{x}(A)=\left\{\begin{array}{lll}
1 & \text { if } & x \in A, \\
0 & \text { if } & x \notin A .
\end{array}\right.
$$

Presented to the Society, August 28, 1963; received by the editors July 30, 1963 and, in revised form, September 13, 1963. 
In order to understand the results we obtain here, it will be necessary to summarize some of the results in [6] and [7].

In [6] Spitzer showed that if the recurrence condition(1)

$$
\sum_{n=1}^{\infty} \frac{P\left(S_{n} \leqq 0\right)}{n}=\infty
$$

holds, then there is a unique $\sigma$-finite measure $\pi(\cdot)$ on the Borel sets of $[0, \infty)$ such that $\pi(\{0\})=1$, and for any Borel set $A \in[0, \infty)$, we have

$$
\pi(A)=\int_{0-}^{\infty} P_{1}(x ; A) \pi(d x) .
$$

Moreover,

$$
\pi(A)=\sum_{n=1}^{\infty}{ }_{0} P_{n}(0 ; A) .
$$

In addition, he also showed that if either the $X_{n}$ have a symmetric distribution or $E X_{1}=0, E X_{1}^{2}<\infty$, then if $A=[0, x]$, we have

$$
\lim _{n \rightarrow \infty} \frac{P_{n}(0 ; A)}{P_{n}(0 ;\{0\})}=\pi(A) \text {. }
$$

Moreover,

$$
P_{n}(0,\{0\}) C \sqrt{ }(n) \rightarrow 1,
$$

where $C$ is an explicitly determined constant. (See (2.18).)

These results of Spitzer lead one to inquire whether or not (1.4) is valid only under the condition (1.2). Our principal goal will be to show that this question can be answered in the affirmative by means of the following

THEOREM 1. If (1.2) holds, then for any bounded Borel set $A \subset[0, \infty)$, any nonnegative $x, y$, and any fixed integer $m$, we have

$$
\lim _{n \rightarrow \infty} \frac{P_{n+m}(x ; A)}{P_{n}(y ;\{0\})}=\pi(A) .
$$

Regarding (1.5), we have the following

(1) It is not difficult to show that when condition (1.2) holds we have, for any Borel set $A$ such that $P_{n}(0 ; A)>0$ for some $n$, that $P\left(T_{n} \in A i . o . \mid T_{0}=x\right)=1$ and, conversely, if $P\left(T_{n}=0\right.$ i.o. $\left.\mid T_{0}=0\right)=1$ then condition (1.2) holds. Thus (1.2) is a necessary and sufficient condition for the $T_{n}$ process to be "recurrent." In particular (1.2) holds if $E\left|X_{1}\right|<\infty$ and $E X_{1} \leqq 0$. 
THEOREM 2. If

$$
\lim _{n \rightarrow \infty} n^{-1} \sum_{k=1}^{n} P\left(S_{k}>0\right)=p(2)
$$

for $0 \leqq p<1$, then

$$
\lim _{n \rightarrow \infty} P_{n}(0 ;\{0\}) \Gamma(1-p) n^{p} L^{-1}(n)=1,
$$

where $L(x)$ is a slowly varying function of known form.

As a corollary of Theorem 1 we shall obtain the following result, which is of some interest in its own right:

Let $V$ be the first passage time for the sums $S_{n}^{\prime}$ to the interval $(0, \infty)$; then for $x \geqq 0$, we have

$$
\lim _{n \rightarrow \infty} \frac{P\left(V>n+m \mid S_{0}^{\prime}=-x\right)}{P\left(V>n \mid S_{0}^{\prime}=0\right)}=\pi([0, x]),
$$

provided (1.2) holds.

In [6] and [7] Spitzer showed also that when $X_{n}$ has density $k(x)$ then every nondecreasing, right continuous solution $F(x)$ of the Wiener-Hopf equation,

$$
F(x)=\int_{0^{-}}^{\infty} k(x-y) F(y) d y,
$$

induces a measure $\pi$ satisfying (1.3) and conversely, every such measure gives rise to a nondecreasing, right continuous solution $F(x)$ of (1.10). Thus, under condition (1.2) there is (modulo a constant) only one such solution $F(x)$. Moreover, if we let $F_{0}(x)$ be an arbitrary probability distribution on the nonnegative axis, and we define functions $F_{n}(x)$ by the relation,

$$
F_{n+1}(x)=\int_{0^{-}}^{\infty} k(x-y) F_{n}(y) d y .
$$

Then (as was pointed out by Spitzer in [7]) it is easy to verify that

$$
F_{n}(x)=\int_{0^{-}}^{\infty} P_{n}(y,[0, x]) d F_{0}(y) .
$$

Consequently, our results show that condition (1.2)alone suffices to guarantee that the iterates (1.11) converge to the unique nondecreasing, right continuous solution of (1.10) (with $F(0)=1$ ) in the following manner:

(2) It is easy to verify that (1.7) implies that (1.2) holds. 


$$
\lim _{n \rightarrow \infty} \frac{F_{n}(x)}{F_{n}(0)}=F(x) \text {. }
$$

2. Proofs. In this section, variables $x, y$ will always denote points on the nonnegative axis while sets $A, B$ will be Borel subsets of $[0, \infty)$. We commence our investigation with the following known result:

Lemma 2.1. If $A=[0, y]$ then

$$
P_{n}(x ; A)=P\left(M_{n-1}^{+} \in A ;\left(S_{n}+x\right)^{+} \in A\right) .
$$

Proof. By induction on $n$ it can readily be verified that

$$
T_{n}=\operatorname{Max}\left\{0, S_{n}+T_{0}, \operatorname{Max}\left(X_{n}, X_{n}+X_{n-1}, \cdots, X_{n}+\cdots+X_{2}\right)\right\} .
$$

Consequently,

$$
P_{n}(x ; A)=P\left[\left(S_{n}+x\right)^{+} \leqq y, \operatorname{Max}\left(X_{n}, X_{n}+X_{n-1}, \cdots, X_{n}+\cdots+X_{2}\right) \leqq y\right]
$$

But as the $X_{n}$ are independent and identically distributed we have that the right-hand side of (2.2) and (2.1) are equal.

The next lemma is basic to the proof of Theorem 1.

Lemma 2.2. If condition (1.2) is satisfied and $A=[0, y]$ then

$$
\lim _{n \rightarrow \infty} \frac{P_{n+1}(x ; A)}{P_{n}(0 ; A)}=1 \text {. }
$$

Proof. A theorem of E. S. Andersen [1] (a simple proof can be found in [3]) asserts for $|t|<1$ we have

$$
\sum_{n=0}^{\infty} P\left(M_{n}^{+}=0\right) t^{n}=\exp \sum_{k=1}^{\infty} P\left(S_{k} \leqq 0\right) t^{k} / k .
$$

Thus, when condition (1.2) holds, we have

$$
\sum_{n=0}^{\infty} P\left(M_{n}^{+}=0\right)=\infty
$$

and since $P\left(M_{n}^{+}=0\right)$ is monotone in $n$, we must have $P\left(M_{n}^{+}=0\right)>0$ for all $n$. Moreover, it is easily shown (see, e.g., [5]) that if (1.2) holds, then $\operatorname{Min}\left(S_{1}, S_{2}, \cdots, S_{n}\right) \rightarrow-\infty$ with probability one. Consequently,

$$
P\left(S_{i}>-x \text { for all } i>0\right)=0 \text {. }
$$

Now

$$
P\left(T_{n} \neq 0 \text { for all } n>0 \mid T_{0}=x\right)=P\left(S_{i}>-x \text { for all } i\right)
$$

and thus

$$
\sum_{n=1}^{\infty}{ }_{0} P_{n}(x ;\{0\})=1
$$


From Lemma (2.1) we have that $P_{n}(0 ; A)$ is monotone in $n$, and thus from the estimate

we obtain

$$
P_{n+1}(x ; A) \geqq \sum_{k=1}^{n}{ }_{0} P_{k}(x ;\{0\}) P_{n+1-k}(0 ; A),
$$

$$
P_{n+1}(x ; A) \geqq P_{n}(0 ; A) \sum_{k=1}^{n}{ }_{0} P_{k}(x ;\{0\}) .
$$

Since $P_{n}(0, A) \geqq P_{n}(0,0)>0$ we have $P_{n}(0 ; A)>0$ for all $n$. Thus, from (2.6) and (2.5) we have

$$
\lim _{n \rightarrow \infty} \frac{P_{n+1}(x ; A)}{P_{n}(0 ; A)} \geqq 1 .
$$

On the other hand, Lemma (2.1) shows $P_{n+1}(x ; A) \leqq P_{n}(0 ; A)$, and thus

$$
\limsup _{n \rightarrow \infty} \frac{P_{n+1}(x ; A)}{P_{n}(0 ; A)} \leqq 1 .
$$

Combining (2.7) and (2.8), we obtain (2.3).

We may now prove Theorem 1 . We first establish the special case for $m=0$, $x=0$, and $y=0$.

LEMMA 2.3. If condition (1.2) holds and $A$ is any bounded Borel set, then

$$
\lim _{n \rightarrow \infty} \frac{P_{n}(0 ; A)}{P_{n}(0 ;\{0\})}=\pi(A),
$$

where

$$
\pi(A)=\sum_{n=1}^{\infty}{ }_{0} P_{n}(0 ; A)
$$

Proof. We have that

$$
\begin{aligned}
\frac{P_{n}(0 ; A)}{P_{n}(0 ;\{0\})} & =\sum_{k=1}^{m} \frac{P_{n-k}(0 ;\{0\})}{P_{n}(0 ;\{0\})}{ }_{0} P_{k}(0 ; A)+\sum_{k=m+1}^{n} \frac{P_{n-k}(0 ;\{0\})}{P_{n}(0 ;\{0\})}{ }_{0} P_{k}(0 ; A) \\
& =G_{1}(m, n ; A)+G_{2}(m, n ; A) .
\end{aligned}
$$

It is easily seen from Lemma 2.2 that

$$
\lim _{m} \lim _{n} G_{1}=\pi(A),
$$

and thus (2.9) will be proved provided we can show that

$$
\lim _{m} \lim _{n} G_{2}=0(3) \text {. }
$$

Now if $A=\{0\},(2.11)$ is evident. Suppose

$\left({ }^{3}\right)$ This procedure for establishing a ratio limit theorem was used by Orey [4] in the investigation of irreducible, null recurrent Markov chains with a denumerable state space. 
We have

$$
A=(a, b], \quad \text { where } b-a<\delta .
$$

$$
\begin{aligned}
{ }_{0} P_{n+m}(0 ;\{0\}) & \geqq \int_{a^{+}}^{b}{ }_{0} P_{n}(0 ; d x){ }_{0} P_{m}(x ;\{0\}) \geqq{ }_{0} P_{n}(0 ; A) \inf _{a<x \leqq b}{ }_{0} P_{m}(x ;\{0\}) \\
& \geqq{ }_{0} P_{n}(0 ; A) P\left(S_{m} \leqq-b, S_{i}>-a, 1 \leqq i<m\right) .
\end{aligned}
$$

Now if $\delta$ is sufficiently small, then for some $m$, say $m_{0}$, we must have (by (2.4)) that

and thus

$$
\alpha=P\left(S_{m_{0}} \leqq-b, S_{i}>-a, 1 \leqq i<m_{0}\right)>0,
$$

$$
{ }_{0} P_{n}(0 ; A) \leqq \alpha^{-1}{ }_{0} P_{n+m_{0}}(0 ;\{0\}) \text {. }
$$

But

$$
\begin{aligned}
G_{2}\left(m+m_{0}, n+m_{0},\{0\}\right) & =\sum_{l=m+m_{0}+1}^{n+m_{0}} \frac{P_{n+m_{0}-l}(0 ;\{0\})}{P_{n+m_{0}}(0 ;\{0\})}{ }_{0} P_{l}(0,\{0\}) \\
& =\frac{P_{n}(0,\{0\})}{P_{n+m_{0}}(0,\{0\})} \sum_{k=m+1}^{n} \frac{P_{n-k}(0,\{0\})}{P_{n}(0,\{0\})}{ }_{0} P_{m_{0}+k}(0,\{0\}),
\end{aligned}
$$

and thus by (2.12) we have

$$
\begin{aligned}
G_{2}(m, n,(a, b]) & \leqq \alpha^{-1} \frac{P_{n+m_{0}}(0,\{0\})}{P_{n}(0,\{0\})} G_{2}\left(m+m_{0}, n+m_{0},\{0\}\right) \\
& \leqq \alpha^{-1} G_{2}\left(m+m_{0}, n+m_{0},\{0\}\right) .
\end{aligned}
$$

Consequently, (2.11) holds for $A=(a, b]$ with $b-a$ sufficiently small. Finally, as ${ }_{0} P(0,$.$) is an additive set function, and as any bounded A$ is contained in a finite disjoint union of such intervals (and $\{0\}$ perhaps), each of length $\leqq \delta$ for any positive $\delta$, we have that (2.11) holds for every bounded $A$. This establishes the lemma.

To complete the proof of Theorem 1 we first observe that if $A$ is any interval of the type $[0, y]$ then by Lemma (2.2) and equation (2.9) we have

$$
\lim _{n \rightarrow \infty} \frac{P_{n+m}(x, A)}{P_{n}(0,\{0\})}=\lim _{n \rightarrow \infty} \frac{P_{n+m}(x, A)}{P_{n}(0, A)} \frac{P_{n}(0, A)}{P_{n}(0,\{0\})}=\pi(A)
$$

and thus (1.6) holds for all such intervals. Now for any $A$ we have

$$
\begin{aligned}
\frac{P_{n+m}(x, A)}{P_{n}(0,\{0\})}= & \frac{\sum_{k=1}^{r} P_{k}(x,\{0\}) P_{n+m-k}(0, A)}{P_{n}(0,\{0\})} \\
& +\left\{\frac{\sum_{k=r+1}^{n+m-1}{ }_{0} P_{k}(x,\{0\}) P_{n+m-k}(0, A)}{P_{n}(0,\{0\})}+\frac{{ }_{0} P_{n+m}(x, A)}{P_{n}(0,\{0\})}\right\}=H_{1}+H_{2} .
\end{aligned}
$$


From (2.9) and (2.5) we have that for any bounded $A$,

$$
\lim _{r \rightarrow \infty} \lim _{n \rightarrow \infty} H_{1}=\pi(A)
$$

and thus to finish the proof we must show

$$
\lim _{r \rightarrow \infty} \lim _{n \rightarrow \infty} H_{2}(A)=0
$$

for all bounded $A$. If $A$ is an interval $[0, y]$, then, by what was just shown above, this must be true and since $A \subset[0, y]$ for some $y \geqq 0$, we have

$$
H_{2}(A) \leqq H_{2}([0, y])
$$

for some $y$ and thus the above assertion is true for any bounded $A$. This then completes the proof of Theorem 1 .

As an immediate consequence of Theorem 1 we have the following extension of Lemma 2.2.

Corollary 2.4. If $A$ is bounded and if $A$ can be "reached" from 0 (i.e., if for some $\left.n, P_{n}(0, A)>0\right)$, then for any fixed integer $m$

$$
\lim _{n \rightarrow \infty} \frac{P_{n+m}(x, A)}{P_{n}(y, A)}=1 .
$$

COROllary 2.5. Let condition (1.2) hold, and define $V$ by

$$
V= \begin{cases}\inf \left\{n: S_{n}>0\right\} & \text { if for some } n \text { we have } S_{n}>0, \\ \infty & \text { otherwise. }\end{cases}
$$

Then for each $x \geqq 0$, we have

$$
\lim _{n \rightarrow \infty} \frac{P\left(V>n+m \mid S_{0}^{\prime}=-x\right)}{P\left(V>n \mid S_{0}^{\prime}=0\right)}=\pi\{[0, x]\} .
$$

Proof. We have

$$
P\left(V>n \mid S_{0}^{\prime}=-x\right)=P\left(M_{n}^{+} \leqq x\right)=P\left(T_{n} \leqq x \mid T_{0}=0\right),
$$

and (2.14) follows at once from Lemma 2.3.

Corollary 2.6. Let

$$
F(x)=\pi\{[0, x]\}, F_{0}(x)=P\left(T_{0} \leqq x\right), F_{n}(x)=\int_{0^{-}}^{\infty} P_{n}(y ;[0, x]) d F_{0}(y) .
$$

Suppose condition (1.2) holds, and $X_{n}$ has density $k(x)$; then

$$
\lim _{n \rightarrow \infty} \frac{F_{n}(x)}{F_{n}(0)}=F(x),
$$

and $F(x)$ is the unique right continuous, nonnegative, nondecreasing solution of $(1.10)$ with $F(0)=1$. 
Proof. As mentioned in the introduction, $F(x)$ is the unique solution to (1.10) with the above properties, and all we need show is that (2.15) holds. Set $A=[0, x]$, and note that by Lemma 2.3 we have for any $z \geqq 0$ that

$$
\lim _{n \rightarrow \infty} \frac{P_{n}(z ; A)}{F_{n}(0)}=F(x) \text {. }
$$

But Lemmas 2.1 and 2.2 show that

$$
\frac{P_{n}(z ; A)}{F_{n}(0)} \leqq \frac{P_{n-1}(0, A)}{F_{n}(0)} \rightarrow F(x)
$$

and thus $P_{n}(z ; A) / F_{n}(0) \leqq C$ (independent of $z$ ). Hence, by bounded convergence we have

$$
\lim _{n \rightarrow \infty} \frac{F_{n}(x)}{F_{n}(0)}=\lim _{n \rightarrow \infty} \int_{0}^{\infty} \frac{P_{n}(z ; A)}{F_{n}(0)} d F_{0}(z)=F(x) .
$$

Next we establish Theorem 2.

If (1.7) holds, then a theorem of Lamperti [2] shows that

$$
1-E t^{V}=(1-t)^{p} L\left(\frac{1}{1-t}\right),
$$

where $L(x)$ is a slowly varying function. However, by a theorem of E. S. Andersen [1] (a simple proof may be found in [3]) we have

and thus

$$
1-E t^{V}=\exp \left(-\sum_{k=1}^{\infty} P\left(S_{k}>0\right) t^{k} / k\right)
$$

$$
\begin{aligned}
L\left(\frac{1}{1-t}\right) & =(1-t)^{-p}\left[1-E t^{\nu}\right]=\exp \left(p \sum t^{k} / k\right) \exp \left(-\Sigma P\left(S_{k}>0\right) t^{k} / k\right) \\
& =\exp \left(-\Sigma\left[p-P\left(S_{k}>0\right)\right] t^{k} / k\right) .
\end{aligned}
$$

Now, clearly

and thus

$$
P\left(T_{n}=0 \mid T_{0}=0\right)=P\left(M_{n}^{+}=0\right)=P(V>n),
$$

$$
\sum_{n=0}^{\infty} P\left(T_{n}=0 \mid T_{0}=0\right) t^{n}=(1-t)^{p-1} L\left(\frac{1}{1-t}\right) .
$$

Karamata's theorem now gives

$$
\sum_{k=0}^{n} P\left(T_{k}=0 \mid T_{0}=0\right) \sim \frac{n^{1-p} L(n)}{\Gamma(2-p)} .
$$

But since $P\left(T_{n}=0 \mid T_{0}=0\right)$ is monotone in $n$, it is permissible to conclude from the above that 


$$
P\left(T_{n}=0 \mid T_{0}=0\right) \sim \frac{n^{-p} L(n)}{\Gamma(1-p)},
$$

which completes the proof.

NotE. In the special case of $E X_{1}=0, E X_{1}^{2}<\infty$, Spitzer [7] established the important result,

$$
\lim _{t \rightarrow 1} L\left(\frac{1}{1-t}\right)=\exp \left(\sum_{k=1}^{\infty}\left(\frac{1}{2}-P\left(S_{k}>0\right)\right)\right)=C .
$$

In conclusion, let us note that the representation

$$
\int_{0^{-}}^{\infty} e^{-\lambda x} \pi(d x)=\exp \left(\sum_{k=1}^{\infty} E\left(e^{-\lambda S_{k}} ; S_{k}>0\right) k^{-1}\right),
$$

which was shown by Spitzer to be valid for the cases investigated by him, remains valid just under assumption (1.2). The same argument as used by Spitzer in [6] suffices to establish (2.16) in general.

\section{REFERENCES}

1. E. S. Andersen, Fluctuation of sums of random variables. II, Math. Scand. 2 (1954), 195-223.

2. J. Lamperti, Some limit theorems for stochastic processes, J. Math. Mech. 7 (1958), 433-450.

3. S. C. Port, Elementary probability approach to fuctuation theory, J. Math. Anal. Appl. 6 (1963), 109-151.

4. S. Orey, Strong ratio limit property, Bull. Amer. Math. Soc. 67 (1961), 571-574.

5. F. Spitzer, A combinatorial lemma and its application to probability theory, Trans. Amer. Math. Soc. 82 (1956), 323-339.

6. - The Wiener-Hopf equation whose kernel is a probability density, Duke Math. J. 24 (1957), 327-344.

7. - A Tauberian theorem and its probability interpretation, Trans. Amer. Math. Soc. 94 (1960), 150-169.

The Rand Corporation,

Santa Monica, California 